\title{
Management of blood donors whose donations are repeatedly falsely positive by the HIV antibody screening test
}

\author{
H I Atrah, J V Parry, D Gough, J Tosswill, F A Ala
}

\begin{abstract}
Since 1985, over 1800000 donations have been screened by the West Midlands Regional Blood Transfusion Service for antibody to HIV. Twelve regular donors gave three or more donations that were alternatingly positive and negative in the screening test, but not confirmed to be HIV positive by supplementary testing. Extensive investigation of six of these donors, including the polymerase chain reaction (PCR), failed to confirm HIV infection. The donors were reassured but, nevertheless, retired to comply with the guidelines of the National Blood Transfusion Service. These findings indicate that, for UK donors, ambiguous serological findings are unlikely to reflect HIV infection. On the rare occasions where serological results are particularly ambiguous, PCR testing of donors' blood may be helpful.

(f Clin Pathol 1995;48:865-867)
\end{abstract}

Keywords: HIV, ELISA, PCR, blood donors.

According to the policy of the United Kingdom Blood Transfusion Service for screening for HIV, a donation that tests repeatedly positive by the screening test should be discarded after an aliquot has been sent to a HIV Reference Centre. ${ }^{1}$ If the confirmatory tests prove to be positive, the donor is counselled and retired from the panel. However, should the confirmatory tests yield a negative or equivocal result, the donor's record at the Regional Blood Transfusion Centre is to be "flagged" so that, although the subsequent donation is also discarded, it is retested by the screening and confirmatory tests. The following outcomes of these tests are possible: if both tests are negative, the donor is reinstated; if the confirmatory test is positive, the donor is counselled and retired; if the screening test remains positive with an equivocal or negative confirmatory test, the donor is retired. The last possibility is the least satisfactory outcome. When a donor is retired, it is incumbent upon the Transfusion Centre to offer an easily comprehensible explanation especially as the reason for retirement is related to the possibility of HIV infection. Donors with equivocal supplementary tests may, after initial counselling, legitimately be referred for further investigation in an appropriate outpatient clinic. However, those with repeatedly positive screening tests and persistently negative confirmatory tests for the infection represent a special group of donors who are not well catered for by the present procedures.

\section{Methods and Results}

Since HIV screening was introduced in October 1985, over 1800000 donations have been tested at the West Midlands Regional Blood Transfusion Centre by several generations of Wellcozyme anti-HIV kits. Repeatedly positive samples are tested at the centre using the Serodia particle agglutination test for antibodies to HIV. During this period, 12 blood donors were identified with a minimum of three donations, each of which were repeatedly positive by the HIV screening tests but negative by confirmatory tests. These donors shared an unusual feature: their HIV antibody positive donations were interspersed by at least one HIV negative donation in the screening test. The first six of these donors were retired by letter. Some telephoned to seek further information and were reassured. The other six donors, one man and five women (age range 21 to 50 years) were asked to give further blood samples to investigate an inconsistent test result on their last donation. None suspected the possibility of HIV infection although two donors thought that we had invited them for further tests because we had found that they had leukaemia. Clotted and EDTA blood samples were obtained from each donor. Both samples were sent to the Hepatitis and $\mathrm{Re}$ trovirus Laboratory of the Virus Reference Division of the Central Public Health Laboratory, London.

At the Hepatitis and Retrovirus Laboratory, the serum samples were tested in several enzyme immunoassays (EIAs) including the assay used by the Transfusion Centre (Wellcozyme HIV 1+2, VK55, Murex Diagnostics, Dartford, UK), Enzygnost HIV 1/2 EIA (OWRP 24/25, Behring Diagnostics, Hounslow, UK) 
Summary of serological findings on the repeatedly reactive donors

\begin{tabular}{|c|c|c|c|c|c|c|c|c|}
\hline \multirow[b]{2}{*}{$\begin{array}{l}\text { Patient } \\
\text { No. }\end{array}$} & \multirow[b]{2}{*}{ Sex } & \multirow[b]{2}{*}{$\begin{array}{l}\text { No. of referred } \\
\text { specimens }\end{array}$} & \multirow[b]{2}{*}{$\begin{array}{l}\text { Date of first } \\
\text { reactive specimen }\end{array}$} & \multicolumn{4}{|c|}{ Serological findings on specimens collected for $P C R$} & \multirow[b]{2}{*}{$\begin{array}{l}\text { Western blot Diagnostic } \\
\text { Biotechnology HIV Blot } 2 \cdot 2\end{array}$} \\
\hline & & & & $\begin{array}{l}\text { Date of PCR } \\
\text { specimen }\end{array}$ & $\begin{array}{l}\text { Wellcozyme } e^{a} H I V \\
1+2 O D / C O^{b}\end{array}$ & $\begin{array}{l}\text { Peptide } \mathrm{HIV} 1 / 2 \\
\text { OD/CO }\end{array}$ & $\begin{array}{l}\text { Recombinant }{ }^{d} H I V \\
1 / 2 O D / C O\end{array}$ & \\
\hline $\begin{array}{l}1 \\
2 \\
3 \\
4 \\
5 \\
6\end{array}$ & $\begin{array}{l}F \\
M \\
F \\
F \\
F \\
F\end{array}$ & $\begin{array}{l}5 \\
5 \\
5 \\
5 \\
3 \\
3\end{array}$ & $\begin{array}{l}\text { May } 1991 \\
\text { Dec } 1990 \\
\text { Oct } 1990 \\
\text { May } 1991 \\
\text { Jul } 1991 \\
\text { Apr } 1991\end{array}$ & $\begin{array}{l}\text { Oct } 1992 \\
\text { Nov } 1992 \\
\text { Jan } 1993 \\
\text { Feb } 1993 \\
\text { Feb } 1993 \\
\text { Mar } 1993\end{array}$ & $\begin{array}{r}>9.00 \\
0.68 \\
1.02 \\
4 \cdot 47 \\
0.50 \\
1.47\end{array}$ & $\begin{array}{l}0 \cdot 11 \\
0 \cdot 09 \\
0 \cdot 10 \\
0.09 \\
0 \cdot 47 \\
0 \cdot 37\end{array}$ & $\begin{array}{l}8 \cdot 07 \\
0 \cdot 17 \\
0 \cdot 19 \\
0 \cdot 02 \\
0 \cdot 02 \\
2 \cdot 22\end{array}$ & $\begin{array}{l}\text { gp120 (trace); gp160 }(1+) \\
\text { p24 }(3+) ; \text { p55 (trace) } \\
\text { p24 (1+) } \\
\text { No bands seen } \\
\text { No bands seen } \\
\text { No bands seen }\end{array}$ \\
\hline
\end{tabular}

a Wellcozyme HIV-1 + 2 EIA is the screening test used by Birmingham BTC.

b Optical density/cut off.

'Peptide assay; Behring HIV 1/2 EIA

d Recombinant assay; Biotest HIV 1/2 EIA.

based on synthetic peptide antigens, and an EIA based on "recombinant" antigens, the Biotest Anti-HIV 1/2 Recombinant (807005, Biotest, Solihull, UK). The serum samples were also tested using a western blot (HIV blot $2 \cdot 2$, Diagnostic Biotechnology, Singapore) which incorporates antigens purified from $\mathrm{HIV}$ infected cell culture and a synthetic HIV-2 antigen.

The EDTA blood specimen was divided into two aliquots: one aliquot was processed using a commercial polymerase chain reaction (PCR) kit for the detection of HIV proviral DNA (Amplicor HIV 1, Roche Diagnostics, Welwyn Garden City, UK); from the second aliquot, CD4 positive cells were purified by mixing the blood with anti-CD4 coated particles with ferrous cores (Dynabeads M-450, Dynal, Oslo, Norway). This permitted purification of CD4 positive cells by magnetic separation. DNA was extracted from the purified cells and subjected to nested PCR using SK68/SK69 primers with an appropriate pair of outer primers.

All but one of the serum samples collected at the time the EDTA specimen was taken (for PCR) were reactive in the recombinant based screening EIA used by the Transfusion Centre (table). Two were reactive in the Biotest HIV $1 / 2$ EIA, which is also based on antigens produced by expression of rDNA. All were unreactive, however, when tested by the Behring HIV 1/2 EIA which uses synthetic oligopeptide antigens. Western blotting revealed that three serum samples were anti-HIV negative-that is, no bands were present, and two were anti$\mathrm{HIV}$ indeterminate, giving reactions with gag derived proteins only. One serum gave a reaction on western blotting that would be classified indeterminate by more stringent interpretive criteria ${ }^{2}$ but, according to the World Health Organisation criteria, ${ }^{3}$ which call for reactivity with two env gene products, the pattern could be considered positive.

However, HIV proviral DNA was not detected by either method in any of the donors' EDTA blood specimens. This and the lack of a persisting, confirmed anti-HIV seroconversion during lengthy follow up lead us to conclude that these six donors were not infected with HIV. We invited the donors to attend individually so that it was possible to "explain the nature of the tests we carried out, the results obtained and their implications". Three donors attended for counselling, the others preferred that we inform their general practitioners dir- ectly. After the donors' permission had been obtained, the general practitioners of those who attended the centre were also informed.

All six donors were retired from the panel. They (or their general practitioners) were told that this decision was taken only to comply with National Blood Transfusion Service codes of practice. The negative PCR results gave us the confidence to reassure the donors (and their general practitioners) that they do not have the infection according to "state-of-theart" tests. One donor who attended for counselling and who appeared to have understood what was said to her, requested at a later date for her own general practitioner to be informed about everything we had done, so that he could explain it again to her. The general practitioners of the six donors were informed that, should their patients require an HIV antibody test in the future for any reason (for example, for insurance purposes), the test might produce a false positive result. General practitioners were advised that such a result should be verified by confirmatory tests.

\section{Discussion}

Within a low incidence population, such as the United Kingdom blood donors, discordant serological findings are virtually never associated with HIV infection. We therefore feel that the appropriate response to the remote possibility of HIV seroconversion in a reactive, but unconfirmable, donation is to seek a follow up specimen for further serological investigation. However, in view of the low probability of such reactivity reflecting true HIV infection, follow up samples are probably best obtained on the occasion of the next donation (usually after four to six months) so as not to alarm donors unnecessarily or put unwarranted extra burden on the system and this is our usual practice. Only on the rarest occasion might this lead to a seroconverting donor not having access to early support and counselling and to additional risk to contacts.

For this investigation we asked our donors to give special samples before the next donation was due because they had already made three or more donations with discordant results. While PCR is not indicated as a routine confirmatory test for HIV antibody reactive donations, the selective use of the test is justified for the small proportion of reactive donors whose serum samples give rise to results in supplementary 
tests that are particularly difficult to interpret; the six donors described in this report are examples.

We believe that we owe it to blood donors to investigate their repeatedly positive results extensively despite the added expense and the time required. The reasons for repeatedly false positive results in screening tests are largely unknown, but this should not mean that the donors are left unsure as to whether or not they have the HIV infection with all the difficulties associated with this diagnosis. Donors' tangible expression of concern for others should, if at all possible, not be allowed to become a source of worry for themselves ${ }^{4}$; they deserve reassurance whenever possible.

1 HMSO. Guidelines for the blood transfusion service. 2nd edn. London: HMSO, 1993.

2 Anonymous. Interpretation and use of the western blot assay for serodiagnosis of human immunodeficiency virus type 1 infection. MMWR 1989;38:1-7.

3 Anonymous. Acquired immunodeficiency syndrome (AIDS): Proposed WHO criteria for interpreting results from western blot assays for HIV-1, HIV-2 and HTLV-I/HTLV-II Wkly Epidemiol Rec 1990;65(37):281-3.

4 Perry S, Jacobsberg L, Fishman B. Suicidal ideation and HIV testing. $¥ A M A$ 1990;263:679-82.

\title{
Osteolytic bone lesions in a patient with idiopathic myelofibrosis and bronchial carcinoma
}

\author{
D J Clutterbuck, A E Morrison, C A Ludlam
}

\begin{abstract}
A 59 year old man with longstanding myelofibrosis and previous splenectomy was incidently found to have a large lytic lesion in his left femur which required operative fixation. He had undergone right upper lobectomy for squamous carcinoma of the bronchus five years earlier. Histological analysis of bone reamings showed no evidence of metastatic carcinoma. Osteosclerosis is frequently noted in patients with myelofibrosis but osteolytic lesions are uncommon and may be confused with metastatic malignancy.

( $\mathcal{H}$ Clin Pathol 1995;48:867-868)
\end{abstract}

Keywords: Myelofibrosis, bronchial carcinoma, osteolytic lesions.

\section{Case report}

A 59 year old man with stable myelofibrosis of 33 years duration presented acutely unwell with bilateral pneumonia, congestive cardiac failure and acute renal failure. He required intensive supportive care including mechanical ventilation, diuretics, inotropes, and intravenous broad spectrum antibiotics. Bronchial washings grew enterococci on culture. Pneumococcal antigen was negative. He made a slow recovery. During his recovery, the patient complained of weakness and paraesthesia in his left leg. He was found to have a flaccid paralysis of the left foot with absent ankle jerk and plantar reflex and sensory loss of all modalities below the knee.

An $x$ ray of the left femur showed a lytic lesion occupying the mid and lower third of the shaft of the bone with extensive endosteal scalloping (figure). A bone scan showed increased uptake of the isotope at the diaphysis of the femur consistent with a metastatic malignancy or primary bone tumour. As there was thought to be significant risk of fracture through this lesion, intramedullary nailing of the bone was performed. Reamings taken from the femur were examined histologically and found to contain macrophages, lymphocytes and haemopoietic cells but there was no evidence of malignancy. Following surgery, the patient made a good recovery. Nerve conduction studies showed changes attributed to critical illness polyneuropathy.

His past medical history included splenectomy at the age of 45 for massive splenomegaly after several painful splenic infarcts, and a right upper lobectomy for squamous carcinoma of the bronchus when aged 55 years.

A skeletal survey during this admission showed an abnormality of the bony trabecular pattern in the head of the left humerus, thought to be due to fibrous dysplasia, previously noted in 1960 at which time no abnormality of the left femur was reported. No bone scan was done at the time of lung resection.

Six months later, despite regular post splenectomy prophylaxis with penicillin $\mathrm{V}$, the patient developed a fatal pneumococcal pneumonia and septicaemia. A postmortem examination was not performed.

\section{Discussion}

Idiopathic myelofibrosis is a chronic myeloproliferative disorder characterised by marrow 